\title{
New Approach for Unambiguous High-Resolution Wide-Swath SAR Imaging
}

\author{
Yueguan Lin, ${ }^{1,2}$ Yida Fan, ${ }^{1,2}$ Hai Jiang, ${ }^{3}$ and Wei Wang ${ }^{1,2}$ \\ ${ }^{1}$ National Disaster Reduction Center of China, MCA, Beijing 100124, China \\ ${ }^{2}$ Key Laboratory of Disaster Reduction and Emergency Response Engineering of the MCA, Beijing 100124, China \\ ${ }^{3}$ National Astronomical Observatories, Chinese Academy of Sciences, Beijing 100012, China
}

Correspondence should be addressed to Yueguan Lin; linyueguan@gmail.com

Received 2 December 2013; Accepted 15 March 2014; Published 14 April 2014

Academic Editor: Tat Soon Yeo

Copyright (C) 2014 Yueguan Lin et al. This is an open access article distributed under the Creative Commons Attribution License, which permits unrestricted use, distribution, and reproduction in any medium, provided the original work is properly cited.

\begin{abstract}
The high-resolution wide-swath (HRWS) SAR system uses a small antenna for transmitting waveform and multiple antennas both in elevation and azimuth for receiving echoes. It has the potential to achieve wide spatial coverage and fine azimuth resolution, while it suffers from elevation pattern loss caused by the presence of topographic height and impaired azimuth resolution caused by nonuniform sampling. A new approach for HRWS SAR imaging based on compressed sensing (CS) is introduced. The data after range compression of multiple elevation apertures are used to estimate direction of arrival (DOA) of targets via CS, and the adaptive digital beamforming in elevation is achieved accordingly, which avoids the pattern loss of scan-on-receive (SCORE) algorithm when topographic height exists. The effective phase centers of the system are nonuniformly distributed when displaced phase center antenna (DPCA) technology is adopted, which causes Doppler ambiguities under traditional SAR imaging algorithms. Azimuth reconstruction based on CS can resolve this problem via precisely modeling the nonuniform sampling. Validation with simulations and experiment in an anechoic chamber are presented.
\end{abstract}

\section{Introduction}

As an active microwave remote sensing imaging tool, synthetic aperture radar (SAR) has the unique capability of obtaining abundant electromagnetic information from ground objects throughout the day and night and in all weather conditions. As such, it has been widely used in military reconnaissance, mapping, resource exploration, environmental protection, disaster management, and so forth. Two most important characteristics of modern SAR systemsazimuth resolution and swath coverage-are contradictive in system design [1]. To coordinate this contradiction, highresolution wide-swath (HRWS) SAR is proposed [2-4]. It uses separate small antenna transmitting radar signals, multiple elevation, and azimuth subantennas receiving echoes. Unlike traditional monostatic SAR, HRWS SAR adopts adaptive beamforming technology and displaced phase center antenna (DPCA) technology in elevation and azimuth, respectively.
HRWS SAR system uses multiple elevation antennas and DBF to form a sharp and high gain pattern which follows the direction of echoes in real time. The weighting coefficients for each channel are set as a function of time. This method is called SCORE (scan-on-receive). The direction of arrival (DOA) is calculated based on the vertical slant-range plane acquisition geometry, under the hypothesis of a strict spherical earth model; that is, no topographic height is considered. While, in real acquisition scenarios, characterized by mountains and valleys, displacements exist between the actual DOA and the preset DOA, which will cause pattern loss with respect to the ideal operational conditions [5, 6]. HRWS SAR system adopts DPCA technique which uses multiple apertures in azimuth and acquires, for each transmitted pulse, additional samples along the synthetic aperture. As a result, the pulse repetition frequency (PRF) enables the unambiguous mapping of a wider image swath. To ensure that these azimuth samples are uniformly distributed, there is a rigid restriction posed on its selection of PRF: SAR platform 
moves just one half of its total antenna length between subsequent radar pulses. Such a rigid selection of the PRF is often in conflict with the optimal design of other system parameters. If this rigid condition is not satisfied, there will be periodically nonuniform sampling in azimuth and azimuth ambiguities will appear under traditional imaging algorithms based on matched filter [7, 8].

In this paper, a new approach for unambiguous HRWS SAR imaging is proposed based on compressed sensing (CS). Multiple elevation spatial sampling points are used to estimate the real-time DOA of echoes, so that the displacement caused by acquisition scenarios is eliminated. As the echoes in elevation are limited by the numbers of subantennas, DOA estimation can be achieved effectively based on CS methods. Real-time adaptive DBF is achieved according to the estimated DOA. CS is introduced into azimuth DPCA SAR imaging. Although nonuniform sampling exists in azimuth, the observation model can be precisely constructed according to system and platform parameters. DPCA imaging algorithm based on CS can well recover the target scene and effectively suppress the azimuth ambiguities.

The rest of the paper is organized as follows. In Section 2 overviews of CS are presented. In Section 3, the model of elevation DBF and azimuth DPCA reconstruction are described in detail. Algorithms are validated via simulations and experiments in Section 4. Finally, Section 5 sums up the paper and presents conclusions.

\section{Compressed Sensing}

The theory of compressed sensing (CS) mainly focuses on the perfect reconstruction of the signal which has certain degree of sparsity. Assume that signal $\mathbf{x}\left(\mathbf{x} \in \mathbb{C}^{N}\right)$ is $J$ sparse under the basis $\Psi_{N \times N}$, which indicates that it can be represented by $\Psi$ with only $J(J \ll N)$ nonzero coefficients

$$
\mathbf{x}=\Psi \boldsymbol{\theta}
$$

where $\boldsymbol{\theta}$ is an $N \times 1$ column vector and there are only $J$ nonzero elements in $\boldsymbol{\theta}$. The observation model of CS is expressed as

$$
\mathrm{y}=\Phi \mathbf{x}=\Phi \Psi \theta
$$

where $\Phi$ is the measurement matrix which contains $M \times N$ elements. It is an ill-posed problem to recover $\mathbf{x}$ when $M<N$. However, according to the principle of CS, $\mathbf{x}$ can be exactly recovered by solving an $\ell_{1}$ optimization problem

$$
\widehat{\boldsymbol{\theta}}=\arg \min \|\boldsymbol{\theta}\|_{1} \quad \text { st. } \quad \mathbf{y}=\boldsymbol{\Phi} \boldsymbol{\Psi} \boldsymbol{\theta},
$$

if the sensing matrix $\Theta=\Phi \Psi$ satisfies the restricted isometry property (RIP) condition.

The number of measurements (i.e., the number of elements of $y$ ) should be $M=c J$ for the assurance of perfect reconstruction, in which $c=O(\log (N / J))$ is the oversampling factor determined by the sparsity of target signal (the sparsity is defined by $J / N$ ). If signal $\mathbf{x}$ is nonideally sparse or the measurement $\mathbf{y}$ contains noise, the problem turns into $[9,10]$

$$
\widehat{\boldsymbol{\theta}}=\operatorname{argmin}\|\boldsymbol{\theta}\|_{1} \quad \text { st. } \quad\|\mathbf{y}-\boldsymbol{\Theta} \boldsymbol{\theta}\|_{2}<\varepsilon,
$$

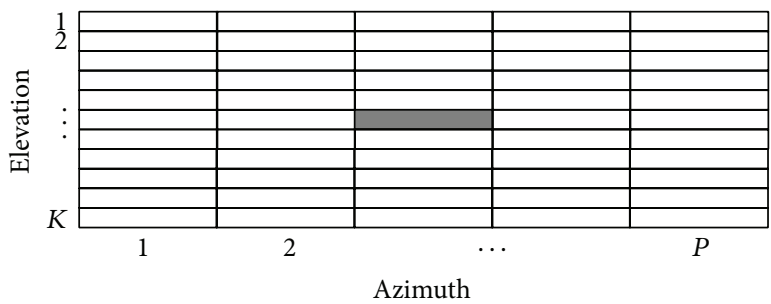

FIGURE 1: Antenna architecture of HRWS SAR.

where $\varepsilon$ describes the error level introduced by the noise or the approximation of $\mathbf{x}$ which is nonideally sparse.

If the sensing matrix $\Theta$ satisfies $J$ order RIP, then, for each $J$ sparse signal $\boldsymbol{\theta}$, there exists the restricted isometry constant (RIC) $\delta_{J} \in(0,1)$ as

$$
\left(1-\delta_{J}\right)\|\boldsymbol{\theta}\|_{2}^{2} \leq\|\Theta \boldsymbol{\theta}\|_{2}^{2} \leq\left(1+\delta_{J}\right)\|\boldsymbol{\theta}\|_{2}^{2}
$$

It is a combinational problem to determine whether a specified matrix satisfies certain order RIP and the RIC cannot be obtained easily. The noncoherence feature is a more widely used criterion which is numerically defined by the maximum cross-correlation coefficient among the columns of $\Theta$

$$
\rho(\boldsymbol{\Theta})=\max _{u \neq v} \frac{|\langle\boldsymbol{\Theta}(u), \boldsymbol{\Theta}(v)\rangle|}{\|\boldsymbol{\Theta}(u)\|_{2}\|\boldsymbol{\Theta}(v)\|_{2}},
$$

where $\boldsymbol{\Theta}(u)$ and $\Theta(v)$ represent the $u$ th and the $v$ th column of $\Theta$, respectively, and $\langle\cdot, \cdot\rangle$ denotes the inner-product of two vectors. In fact, $\rho(\boldsymbol{\Theta})$ equals the maximum value of the nondiagonal elements in the matrix $\mathbf{G}=\boldsymbol{\Theta}^{T} \boldsymbol{\Theta}$ after normalized processing. A larger value of $\rho(\boldsymbol{\Theta})$ indicates the more similar two columns in the sensing matrix, which may cause errors when the reconstruction algorithm tries to locate the support of the signal. The target signal can be recovered exactly if $\boldsymbol{\theta}$ satisfies the following condition [9]:

$$
\rho=\|\boldsymbol{\theta}\|_{0}<\frac{1}{2}\left(1+\frac{1}{\rho(\boldsymbol{\Theta})}\right) .
$$

It has been proved that CS has the potential of reducing the radar raw data volume and simplifying the system complexity. The application of CS combined with imaging radar technology has become the research focus in recent years [11].

\section{HRWS SAR Signal Model Based on CS}

The antenna structure of HRWS system is shown in Figure 1. There are $K$ subantennas in elevation direction and $P$ subantennas in azimuth direction (for simplicity, the values of $K$ and $P$ are set to be the odd numbers). A small antenna in the antenna system center transmits the signal and all the subantennas receive the echoes. The length of transmitting antenna in the elevation direction is designed to be small enough to cover the wide swath. 


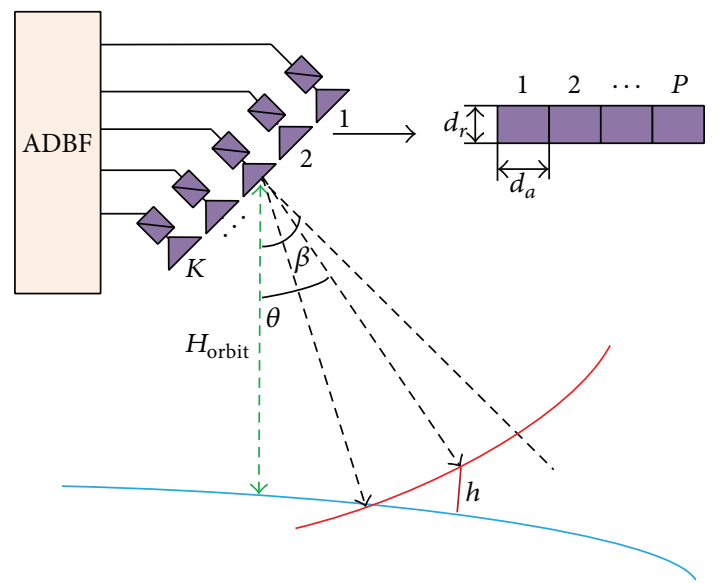

FIGURE 2: Geometry of HRWS SAR elevation adaptive DBF.

3.1. Elevation Signal Model. To increase the antenna gain and reduce the ambiguity, we adopt the digital beam forming (DBF) for the multiple receiving channels to obtain a narrow beam and change the weighting factors of the antenna array instantly to make the beam scan from the near end to the far end within the swath to track and receive the echo of observation terrain. The geometric model of DBF in the elevation direction is shown in Figure 2. After the pulse compression for each echo signal from the multiple channels, the wave direction in each range cell is sparse. According to the CS theory, the direction of the wave can be exactly estimated with few data. In the following, we establish the CS-based elevation signal model. In order to simplify the description, the signal model in a single range cell is considered. The transmitted signal is expressed as

$$
s(\tau)=\operatorname{rect}\left(\frac{\tau}{T}\right) \exp \left(-j 2 \pi f_{c} \tau\right) \exp \left(j \pi K_{r} \tau^{2}\right),
$$

where $\tau$ is the range time, $T$ is the pulse duration time, $K_{r}$ is the frequency modulation rate, and $f_{c}$ is the carrier frequency. Taking the $K$ subantennas (which locate in the center of azimuth direction) for example, and assuming a zero slant look angle, the distance between the target point and the $k$ th $(k=1, \ldots, K)$ subantenna in the elevation direction is

$$
\begin{aligned}
R_{k}= & {\left[R_{(K+1) / 2}^{2}+\left(\left(k-\frac{K+1}{2}\right) d_{r}\right)^{2}\right.} \\
& \left.-2 R_{(K+1) / 2}\left(k-\frac{K+1}{2}\right) d_{r} \sin (\beta-\theta)\right]^{1 / 2},
\end{aligned}
$$

where $R_{(K+1) / 2}$ is the distance between the $(K+1) / 2$ subantenna and the target point. $d_{r}$ is the distance of the adjacent phase centers in the elevation direction. $\beta$ is the angle between the antenna point direction and the vertical direction to earth center. $\theta$ is the angle between the wave direction from target point and its vertical direction to earth center. In case of far-field, $R_{k}$ is written as

$$
\begin{aligned}
R_{k} \approx & R_{(K+1) / 2}-\left(k-\frac{K+1}{2}\right) d_{r} \sin (\beta-\theta) \\
& +\frac{1}{2 R_{(K+1) / 2}}\left(\left(k-\frac{K+1}{2}\right) d_{r}\right)^{2},
\end{aligned}
$$

where the third item can be ignored since it is relatively small compared with the former two items,

$$
R_{k} \approx R_{(K+1) / 2}-\left(k-\frac{K+1}{2}\right) d_{r} \sin (\beta-\theta) .
$$

For the point target in the nearest slant range, the two-way delay in time is

$$
\begin{aligned}
\tau_{k} & =\frac{2 R_{(K+1) / 2}}{c_{0}}-\frac{1}{c_{0}}\left(k-\frac{K+1}{2}\right) d_{r} \sin (\beta-\theta) \\
& =\tau_{0}-\frac{1}{c_{0}}\left(k-\frac{K+1}{2}\right) d_{r} \sin (\beta-\theta),
\end{aligned}
$$

where $c_{0}$ is the light speed and $\tau_{0}$ is the two-way delay in time for the $(K+1) / 2$ th subantenna. As a conclusion, regardless of the electromagnetic loss in the air, the system loss, and the envelope change caused by the platform position, the baseband form of the received signal from the $k$ th subantenna is

$$
\begin{aligned}
r_{k}(t)= & \alpha \cdot \operatorname{rect}\left(\frac{t-\tau_{k}}{T}\right) \\
& \cdot \exp \left(j \pi K_{r}\left(\tau-\tau_{k}\right)^{2}+j 2 \pi f_{c}\left(\tau-\tau_{k}\right)\right),
\end{aligned}
$$

where $\alpha$ is the backscattering coefficients of the point target. After demodulation and range compression, the signal is written as

$$
\begin{aligned}
r_{k}(t)= & \alpha \exp \left(j 2 \pi f_{c} \tau_{0}\right) \cdot \operatorname{sinc}\left(B_{r}\left(\tau-\tau_{k}\right)\right) \\
& \cdot \exp \left(-j \frac{2 \pi}{\lambda}\left(k-\frac{K+1}{2}\right) d_{r} \sin (\beta-\theta)\right),
\end{aligned}
$$

where $\lambda$ is the carrier wavelength. By registration processing,

$$
\begin{aligned}
r_{k}(t)= & \alpha \exp \left(j 2 \pi f_{c} \tau_{0}\right) \cdot \operatorname{sinc}\left(B_{r}\left(\tau-\tau_{0}\right)\right) \\
& \cdot \exp \left(-j \frac{2 \pi}{\lambda}\left(k-\frac{K+1}{2}\right) d_{r} \sin (\beta-\theta)\right) .
\end{aligned}
$$

Thus, for the samples at $t=\tau_{0}$, the compressed signal is expressed as

$$
r_{k}=\alpha \exp \left(-j \frac{2 \pi}{\lambda}\left(k-\frac{K+1}{2}\right) d_{r} \sin (\beta-\theta)\right) .
$$

In the aforementioned formula, the exponential item exp $\left(j 2 \pi f_{c} \tau_{0}\right)$ is included into $\alpha$. Considering the additive noise, the vector form of the multichannel data is expressed as

$$
\mathbf{y}=\alpha \cdot \mathbf{a}(\theta)+\mathbf{e}_{\mathbf{r}}
$$

where $\mathbf{y}, \mathbf{a}(\theta)$, and $\mathbf{e}_{\mathbf{r}}$ are $K \times 1$ vectors. $\mathbf{e}_{\mathbf{r}}$ is the noise vector. $\mathbf{a}(\theta)$ is the point vector which includes the phase information among different channels and has a direct connection with the wave direction. The $k$ th element of $\mathbf{a}(\theta)$ is written as

$$
\mathbf{a}(\theta, k)=\exp \left(j \frac{2 \pi}{\lambda}\left(k-\frac{K+1}{2}\right) d_{r} \sin (\beta-\theta)\right) .
$$

The system may receive echoes from many directions at a single time, which is caused by the range ambiguity 
and folding effect. In order to obtain the correct wave direction, the wave direction range is discretely divided into several angle grids

$$
\theta_{n}=\theta_{0}+n \cdot \Delta \theta, \quad n=1,2, \ldots N,
$$

where $\theta_{0}$ is the wave direction of the near range of the swath and $\Delta \theta$ is the minimum discrete angle step. The expression which contains echo from all the wave directions can be written as

$$
\mathbf{y}=\sum_{n=1}^{N} \alpha_{n} \cdot \mathbf{a}\left(\theta_{n}\right)+\mathbf{e}_{\mathbf{r}} .
$$

In matrix-vector form,

$$
\mathrm{y}=\mathrm{A} \alpha+\mathrm{e}_{\mathbf{r}}
$$

where $\mathbf{A}$ is an $N \times K$ matrix,

$$
\begin{gathered}
\mathbf{A}(n, k)=\exp \left(j \frac{2 \pi}{\lambda}\left(k-\frac{K+1}{2}\right) d_{r} \sin \left(\beta-\theta_{n}\right)\right), \\
\boldsymbol{\alpha}=\left[\begin{array}{llll}
\alpha_{1} & \alpha_{2} & \cdots & \alpha_{N}
\end{array}\right]^{T} .
\end{gathered}
$$

The number of the wave directions is finite and sparse compared with all the directions in real application, which means that the number of nonzero elements in $\boldsymbol{\alpha}$ is far less than $N$. By applying CS, they can be estimated and then the wave direction $\theta_{n}$ can be calculated accordingly. The estimation of $\alpha$ is modeled as

$$
\min (|\widetilde{\boldsymbol{\alpha}}|), \quad \text { st. } \quad\|\mathbf{y}-\mathbf{A} \tilde{\boldsymbol{\alpha}}\|<\left\|\mathbf{e}_{\mathbf{r}}\right\|_{2} .
$$

3.2. Azimuth Signal Model. The HRWS SAR system adopts displaced phase center antenna (DPCA) technology to retrieve the azimuth signal by rearranging the multichannel echoes in the order of equivalent phase center and avoids the Doppler ambiguity caused by limited sampling. However, DPCA requires that SAR platform moves just one half of its total antenna length between subsequent radar pulses. This condition is often difficult to meet in real applications, which makes the arrangement of the equivalent phase center nonuniform. And the azimuth ambiguity emerges when directly processing with the nonuniform azimuth signal. Currently, there are two methods to solve the problem, the phase compensation method and the spectrum reconstruction method. The phase compensation method corrects the phase of the multiaperture data so that it corresponds to single uniform SAR signal. The main disadvantage of this method is the severe impact on the resolution [12]. The spectrum reconstruction method retrieves the unambiguous signal from aliasing Doppler spectrum by solving a serial of filter banks. It can be done in time domain or frequency domain, but the calculation complexity of this algorithm is time-consuming [13].

The CS-based azimuth nonuniform sampling reconstruction method builds the exact observation model by using the system and platform parameters and then achieves the recovery through solving an $\ell_{1}$ norm minimizing problem. Taking the $P$ azimuth subantennas locating in the elevation center of the antenna array, for example, with the ignorance of the influence on the signal amplitude caused by range compression, the echo model of the $p$ th $(p=1, \ldots, P)$ subantenna is

$$
h_{p}(t)=\exp \left(-j \frac{2 \pi}{\lambda}\left(\sqrt{r_{0}^{2}+(v t)^{2}}+\sqrt{r_{0}^{2}+\left(v t-p d_{a}\right)^{2}}\right)\right),
$$

where $r_{0}$ is the nearest slant range, $v$ is the platform velocity, $t$ is the azimuth time, and $d_{a}$ is the length of the receiving subantenna. Let $\Delta t_{0}=1 / P R F$; then the distance that the platform moves within a pulse repetition interval is $\delta_{0}=$ $\Delta t_{0} v$. For the system that has one transmitting channel and $P$ receiving channels, the discrete grid of the observation scene can be set to $\delta_{y}=\delta_{0} / P$ to achieve the high-resolution reconstruction. As a conclusion, assume $\Delta t=\Delta t_{0} / P$; then discrete form of the azimuth samples is

$$
\begin{array}{r}
h_{p}(q)=\exp \left(-j \frac{2 \pi}{\lambda}\left(\sqrt{r_{0}^{2}+(q \cdot v \Delta t)^{2}}\right.\right. \\
\left.\left.+\sqrt{r_{0}^{2}+\left(q \cdot v \Delta t-p d_{a}\right)^{2}}\right)\right), \\
q=1, \ldots, Q .
\end{array}
$$

The azimuth observation model of the $p$ th subantenna is expressed with (26), in which the time interval of the row vector is $\Delta t$, while the time interval of the column vector is $\Delta t P$. Consider

$$
\Phi_{p}=\left[\begin{array}{cccccccc}
h_{p}(1) & 0 & \cdots & 0 & \cdots & 0 & \cdots & 0 \\
h_{p}(P+1) & h_{p}(P) & \cdots & h_{p}(1) & 0 & \ddots & \ddots & 0 \\
\vdots & \vdots & \ddots & \vdots & \ddots & \ddots & \ddots & \vdots \\
h_{p}(Q) & h_{p}(Q-1) & \cdots & h_{p}(Q-P) & \cdots & h_{p}(1) & \ddots & \vdots \\
0 & 0 & \cdots & h_{p}(Q) & \cdots & h_{p}(P+1) & \ddots & h_{p}(1) \\
\vdots & \vdots & \vdots & 0 & \ddots & \ddots & \ddots & \vdots \\
0 & 0 & \cdots & 0 & \cdots & \cdots & 0 & h_{p}(Q)
\end{array}\right] .
$$


Accordingly, the observation matrix $\Phi$ of the whole azimuth observation model should be reorganized from observation matrices of subapertures. After reorganization, the $(k, l)$ elements of $\Phi$ are

$$
\boldsymbol{\Phi}(k, l)=\boldsymbol{\Phi}_{s}(P \cdot\lfloor k / P\rfloor+1, l),
$$

where $\lfloor k / P\rfloor$ is to get the largest integer less than $k / P$ and

$$
s=k-P \cdot\lfloor k / P\rfloor .
$$

After azimuth scene discretization, the reflectivities of scene can be expressed as an $N \times 1$ column vector

$$
\mathbf{x}=\left[\begin{array}{llll}
x_{1} & x_{2} & \cdots & x_{N}
\end{array}\right]^{T} .
$$

If we rearrange the data of each subaperture according to the subsequence of effective phase centers, the measurements can be expressed as follows:

$$
\mathbf{w}=\left[\begin{array}{lllllll}
y_{1}(1) & y_{2}(1) & \cdots & y_{P}(1) & y_{1}(2) & \cdots & y_{P}(N)
\end{array}\right]^{T},
$$

Thus, the observation model can be expressed as

$$
\mathbf{w}=\Phi \mathbf{x}+\mathbf{e}_{\mathbf{a}},
$$

where $\mathbf{e}_{\mathbf{a}}$ is the corresponding additive observation noise vector. Finally, the observed target scene can be reconstructed through solving a constrained $\ell_{1}$ minimization problem

$$
\min (|\widetilde{\mathbf{x}}|), \quad \text { st. } \quad\|\mathbf{w}-\boldsymbol{\Phi} \widetilde{\mathbf{x}}\|<\left\|\mathbf{e}_{\mathbf{a}}\right\|_{2} .
$$

3.3. HRWS SAR Imaging Flowchart. In order to reduce the amount of data satellite transmission, real-time estimation of DOA and digital beamforming are carried out with range compressed echoes from different channels. Only $1 / K$ of data needs to be transmitted to the ground. Azimuth unambiguous reconstruction is achieved on the ground. So, HRWS SAR imaging processing is divided into two parts: onboard realtime processing and ground processing, as shown in Figure 3.

\section{HRWS SAR Simulation}

4.1. Elevation DOA and Adaptive Beamforming. According to the simulation parameters in Table 1, DOA of the echoes from targets at different heights can be calculated. Angular displacements in the presence of topographic height at different slants are illustrated in Figure 4. It can be found that angular displacement reaches $0.1^{\circ}$ to $0.2^{\circ}$ at height of $1 \mathrm{~km}$, and it reaches $0.3^{\circ}$ to $0.4^{\circ}$ at height of $2 \mathrm{~km}$. Angular displacement increases with increasing height of target. SCORE pattern loss in the presence of topographic height can also be calculated as shown in Figure 5. We find that when the target height reaches $2 \mathrm{~km}$ which is a common situation, the pattern loss is close to $2 \mathrm{~dB}$. Therefore, it is necessary to use real-time digital beam forming techniques to estimate the DOA and avoid the pattern loss due to target height.

We consider three targets with the same slant range distance but with different incidence angles, which are $30^{\circ}$,

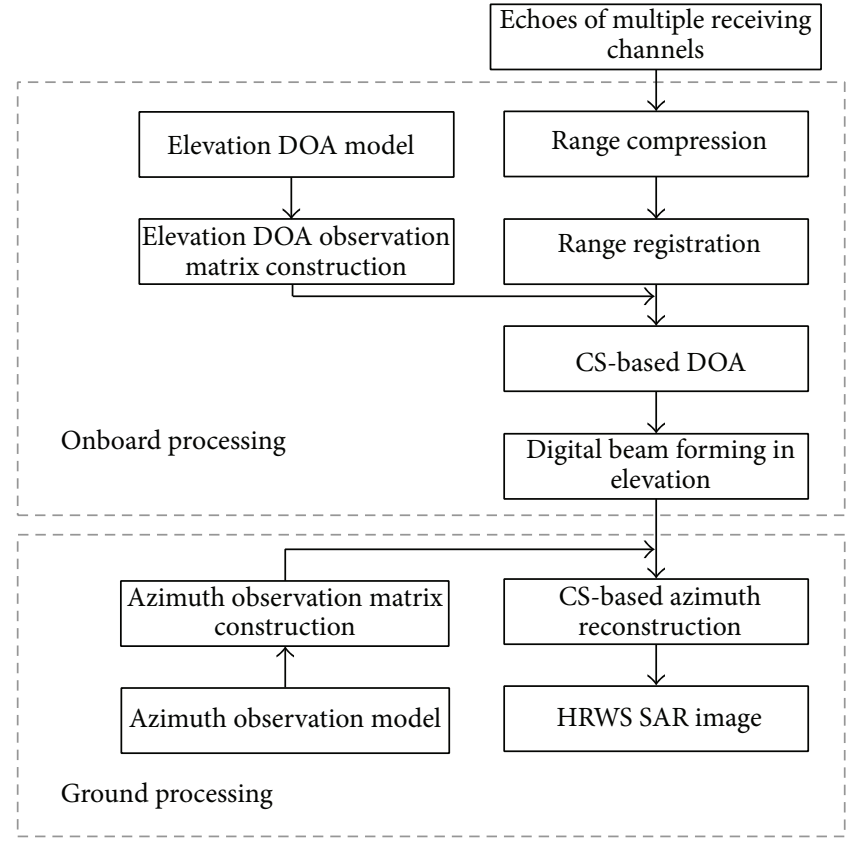

FIgURE 3: Flowchart of unambiguous HRWS SAR imaging.

TABLE 1: Simulation system parameters.

\begin{tabular}{lc}
\hline Parameter & Value \\
\hline Carrier wavelength & $0.03 \mathrm{~m}$ \\
Pulse duration & $50 \mu \mathrm{s}$ \\
Platform height & $560 \mathrm{~km}$ \\
Prf & $1200 \mathrm{~Hz}$ \\
Look angle & {$[29.6,34.9]^{\circ}$} \\
Pulse bandwidth & $200 \mathrm{MHz}$ \\
Transmitting antenna length & $2.5 \mathrm{~m}$ \\
Transmitting antenna height & $0.5 \mathrm{~m}$ \\
Number of receiving antennas & $4 \times 15$ \\
(azimuth $\times$ elevation, $P \times K)$ & $2.5 \mathrm{~m}$ \\
Receiving antenna length & $0.1 \mathrm{~m}$ \\
Receiving antenna height & $7584.1 \mathrm{~m} / \mathrm{s}$ \\
Platform velocity & $4800 \mathrm{~Hz}$ \\
\hline Azimuth effective sampling rate
\end{tabular}

$32^{\circ}$, and $33^{\circ}$, respectively, and the incidence angle space $[29.6,34.9]^{\circ}$ according to the whole swath is divided into 530 subangels evenly with each angle interval being $0.01^{\circ}$. The signals are pure and without noise pollution. Laplace optimization algorithm is adopted for CS reconstruction in our simulation [14]. Results of DOA estimation based on CS are shown in Figure 6. We find that the three targets with different DOA are correctly recovered.

In real acquisition scenarios, we need to estimate DOA at each range sampling time and the number of used samples is decided by the number of subantennas in elevation direction $K$. The number of effective sampling points and SNR of signals after range compression are important factors which 


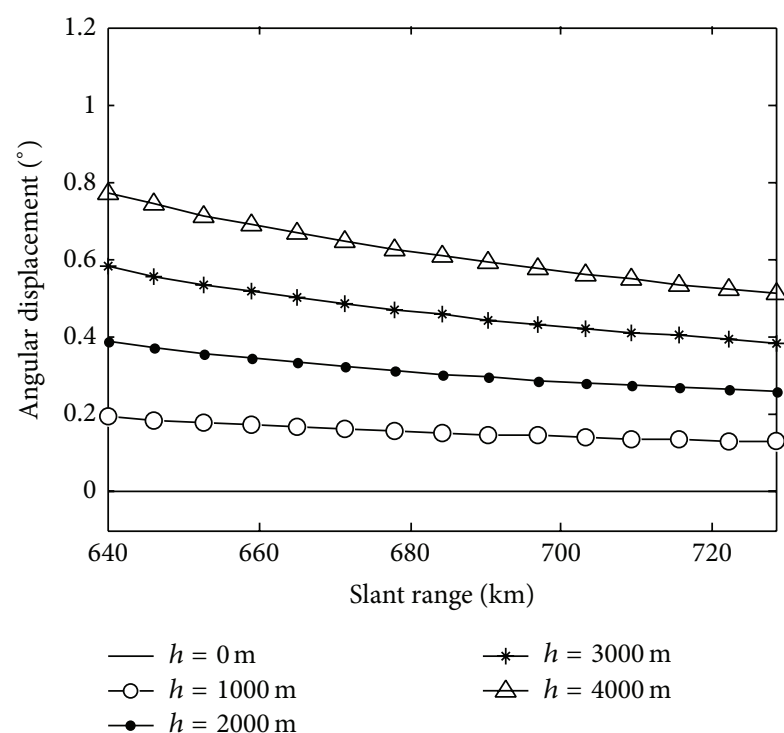

FIGURE 4: Angular displacement in the presence of topographic height.

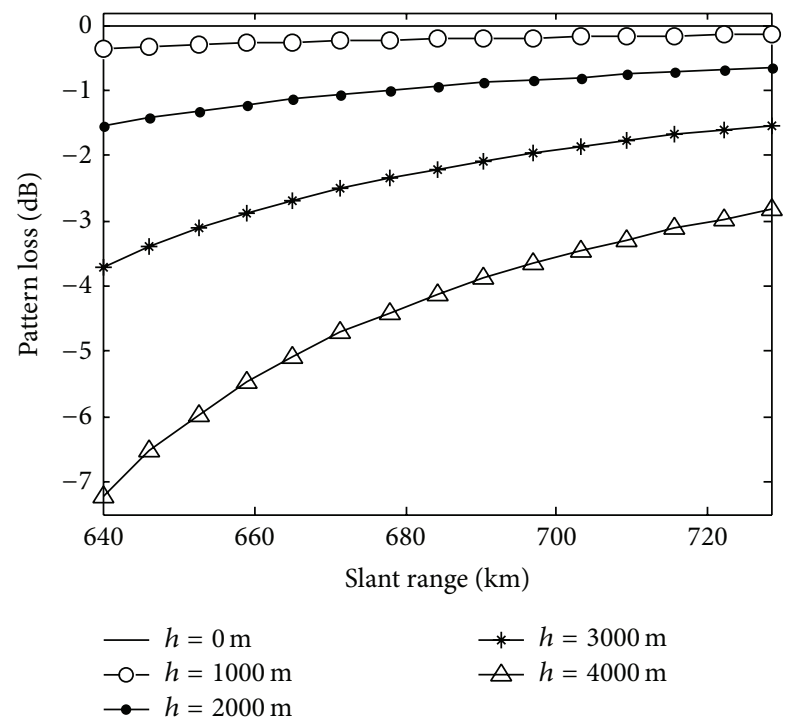

FIGURE 5: SCORE pattern loss in the presence of topographic height.

influence the estimation result. The performance of DOA estimation is calculated empirically via simulation. We randomly set three targets with different topographic height and incident angle for each trail. The trail is declared a success if the sum of the differences between estimated DOA and preset DOA is within the range of $0.1^{\circ}$. The empirical probability of success is determined by repeating the CS reconstruction trials 1000 times and calculating the percentage of success. This percentage of correct DOA estimation is plotted as a function of SNR in Figure 7 (labeled as monoazimuth observation). The results show that the method with monoazimuth sampling can by $100 \%$ correctly estimate DOA only when SNR is higher than $15 \mathrm{~dB}$. This level of SNR for range compressed signal is hard to achieve.

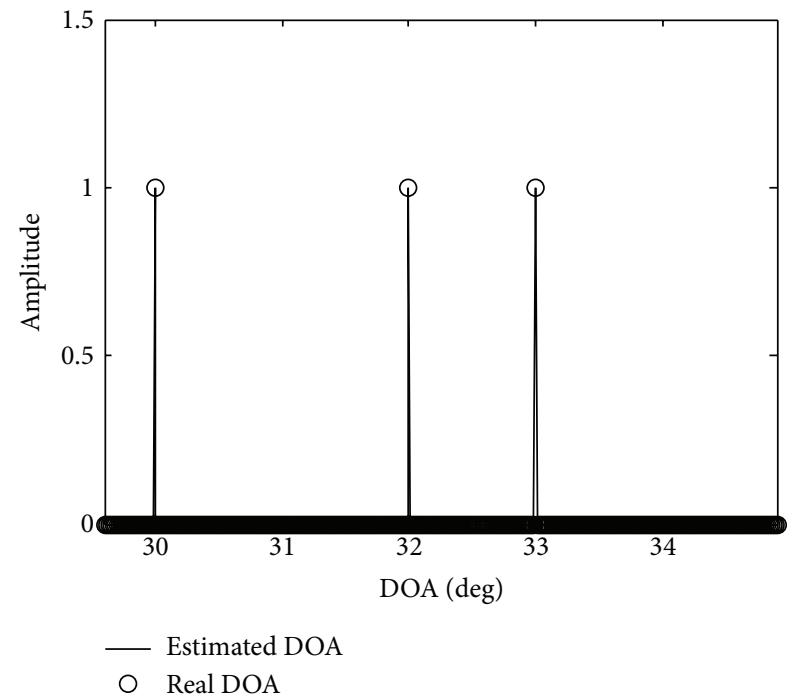

FIGURE 6: Estimation results of DOA based on CS.

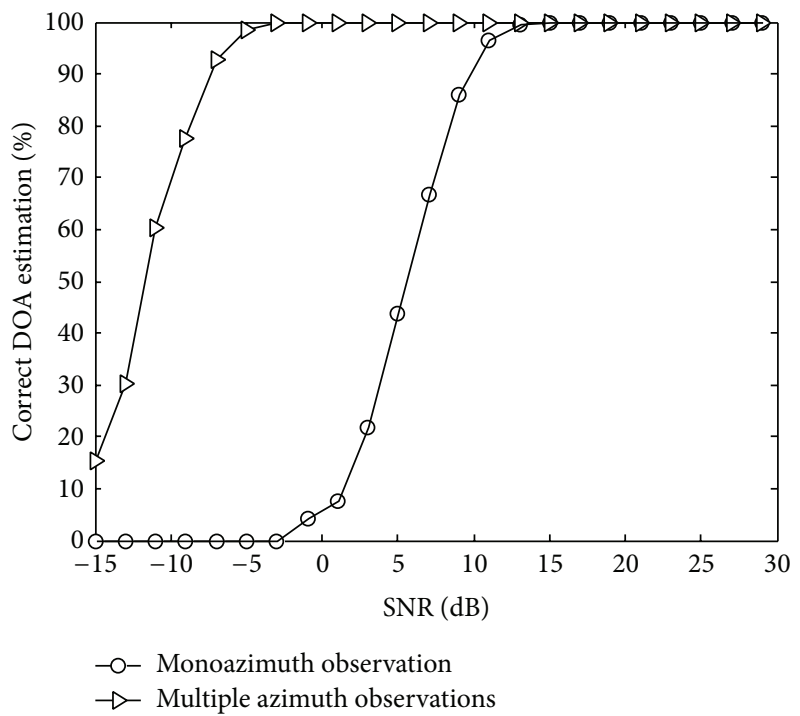

Figure 7: The percentage of correct DOA estimation calculated from 1000 trials as a function of SNR.

If more independent observations are used, the DOA estimation performance will be much improved. As the satellite is moving, observation geometry for the same target at adjacent azimuth time changes. And the satellite echo delay varies because of the existence of range cell migration (RCM), which affects the correct estimation of DOA. In fact, if we adopt nearby azimuth samplings to estimate DOA jointly, it is possible that the error is in the tolerable range. Meanwhile, if target position and amplitude variations caused by RCM exceed the tolerance range, they can be compensated by registration. These variations caused by platform motion can be illustrated by the following simulation in which 100 adjacent azimuth sampling points are adapted. DOA change and RCM against number of azimuth samples are shown in Figures 8 and 9, respectively. We can find that DOA 


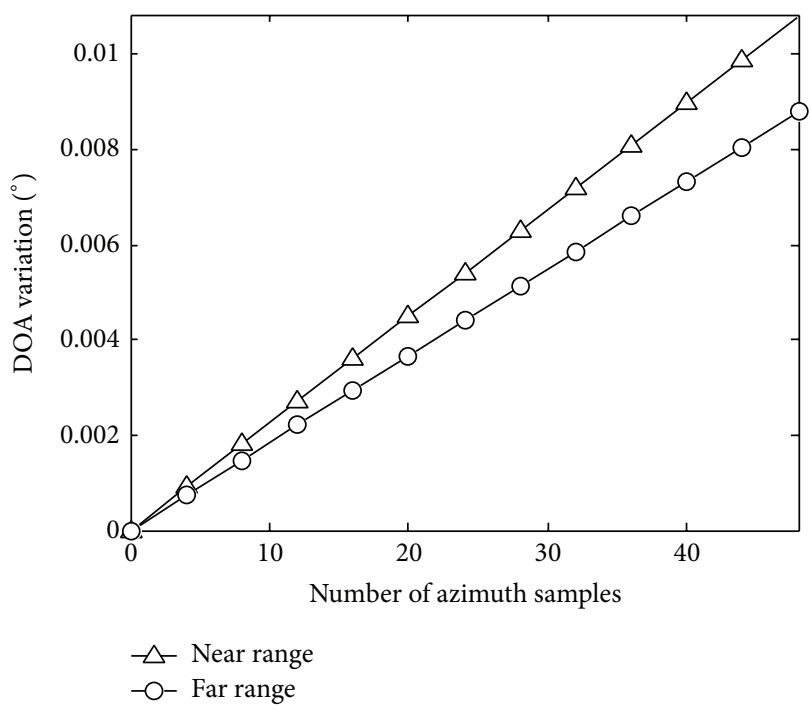

FIGURE 8: DOA variation versus number of azimuth samples.

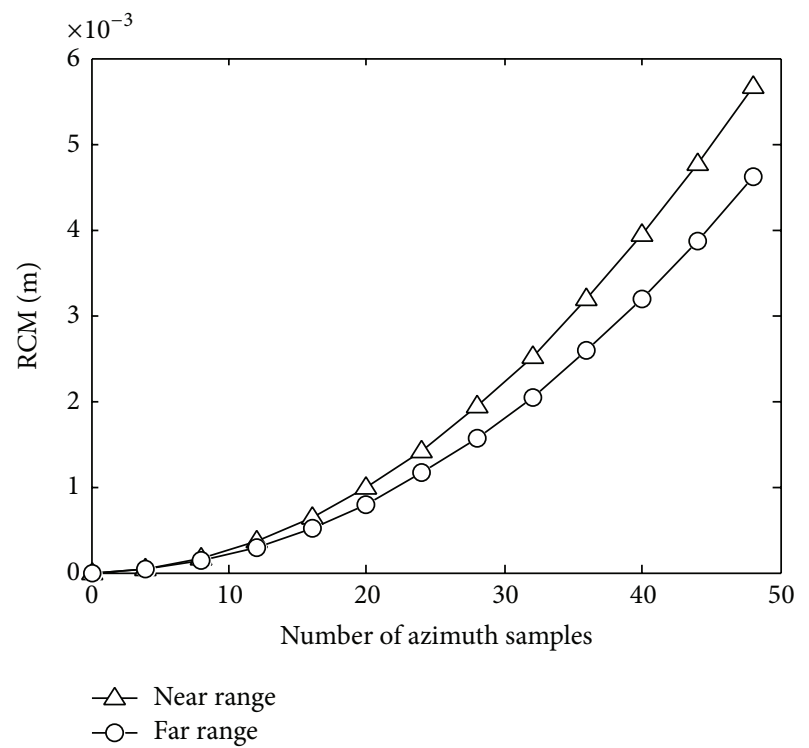

FIGURE 9: RCM versus number of azimuth samples.

changes and RCM is very small and can be ignored in actual processing.

The performance of DOA estimation from multiple azimuth samples is calculated in the same way as monoazimuth sampling. The number of adjacent azimuth samplings is set as 16 , so the number of effective samples for each estimation reach $16 \times K=300$. From results illustrated in Figure 7, we find that joint use of multiple azimuth samples can by $100 \%$ correctly estimate DOA even when SNR is higher than $-3 \mathrm{~dB}$.

4.2. Azimuth Unambiguous Reconstruction. In order to simulate the azimuth reconstruction for HRWS SAR, five point targets are equally spaced in azimuth, and the distance between adjacent target is set as $35 \mathrm{~m}$. Result of traditional

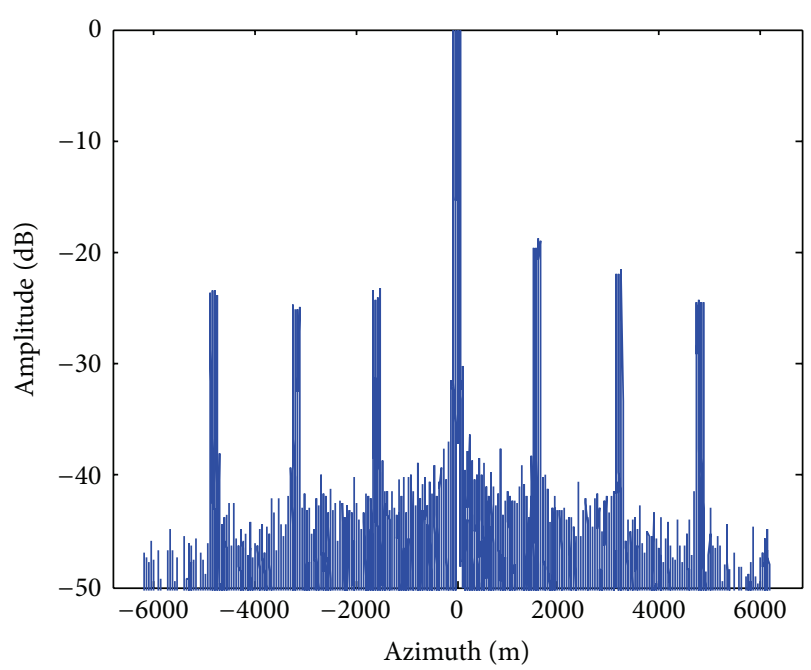

(a) Traditional RDA

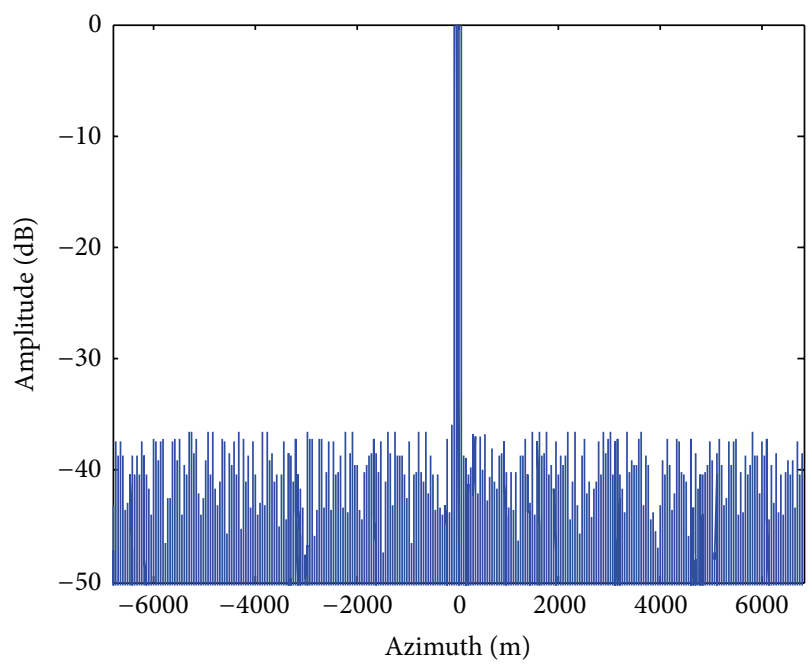

(b) CS imaging algorithm

FIGURE 10: Azimuth slice of imaging results when nonuniform sampling exits in azimuth.

range Doppler (RD) algorithm [15] is shown in Figure 10(a), where azimuth ambiguity reaches to $-19 \mathrm{~dB}$. The CS reconstruction results in azimuth are shown in Figure 10(b). As seen in Figure 10, azimuth ambiguity caused by nonuniform sampling is effectively suppressed by CS methods to a level below $-38 \mathrm{~dB}$.

\section{Experiment in an Anechoic Chamber}

Experiments for azimuth ambiguity suppression using CS is also carried out in an anechoic chamber. Antenna configuration and trihedral corner reflector of this ground-based DPCA SAR system are shown in Figure 11. It has one aperture transmitting stepped frequency and three apertures receiving echoes in azimuth. These four apertures are $3.2 \mathrm{~cm}$ long and tightly arranged. The system parameters are listed in Table 2. 


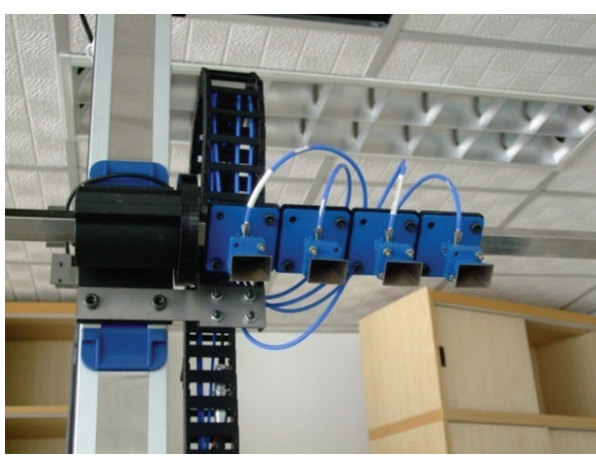

(a) Transmitting and receiving antenna configuration

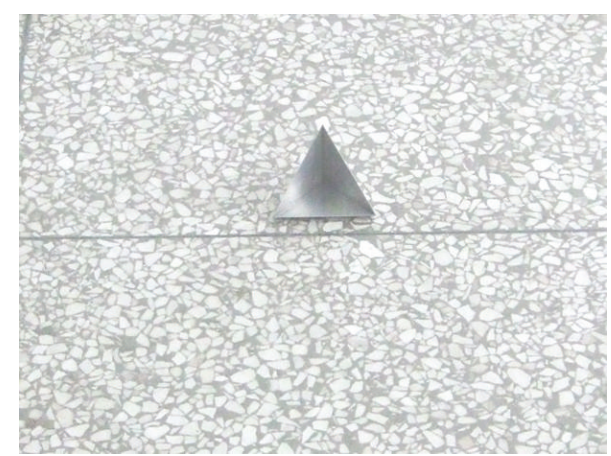

(b) Trihedral corner reflector

FIGURE 11: Antenna configuration and trihedral corner reflector in ground-based DPCA SAR system.

TABLE 2: Ground-based DPCA SAR system parameters.

\begin{tabular}{lc}
\hline Parameters & Values \\
\hline Carrier frequency & $17 \mathrm{GHz}$ \\
Based band width & $1 \mathrm{GHz}$ \\
Stepped frequencies & $2.5 \mathrm{MHz}$ \\
Span in azimuth & {$[-1,1] \mathrm{m}$} \\
Azimuth sampling interval & $0.024 \mathrm{~m}$ \\
Length of subaperture & $0.032 \mathrm{~m}$ \\
\hline
\end{tabular}

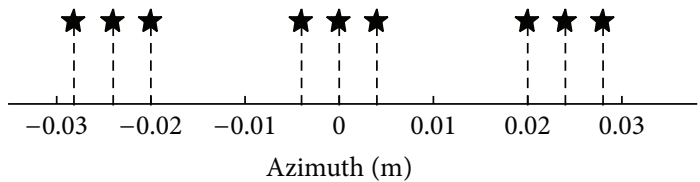

FIGURE 12: Azimuth effective phase centers of ground-based SAR system.

TABLE 3: Comparison of performance for imaging results.

\begin{tabular}{lccc}
\hline & IRW $(\mathrm{m})$ & PSLR $(\mathrm{dB})$ & ISLR $(\mathrm{dB})$ \\
\hline $\begin{array}{l}\text { DPCA SAR imaging with } \\
\text { traditional RD method }\end{array}$ & 0.016 & -15.84 & -8.78 \\
$\begin{array}{l}\text { DPCA SAR imaging with } \\
\text { proposed CS method }\end{array}$ & 0.015 & -13.35 & -11.62 \\
\hline
\end{tabular}

Effective azimuth centers of this DPCA SAR system are shown in Figure 12 and they are nonuniformly distributed. The observed trihedral corner reflector located at the slant range of 2.5 meters. The experimental results with traditional RDA and CS algorithm are shown in Figure 13. We use impulse response width (IRW), peak sidelobe ratio (PSLR), and integrated sidelobe ratio (ISLR) as the performance indexes, and the results are shown in Table 3. It becomes clear that DPCA SAR imaging result with traditional RDA is strongly blurred because the azimuth effective phase centers are nonuniformly spaced. Our proposed CS imaging algorithm can effectively suppress the azimuth ambiguity caused by nonuniform sampling while keeping high azimuth resolution.

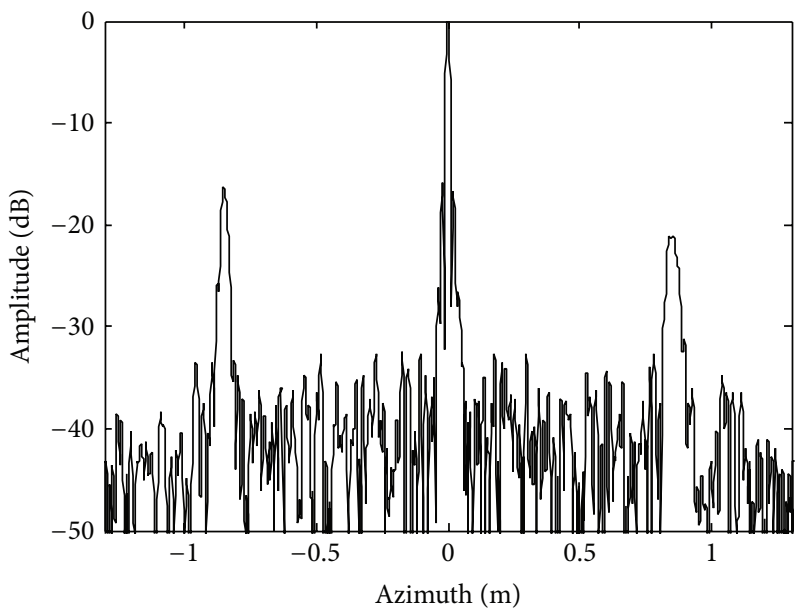

(a) DPCA SAR imaging with traditional RDA

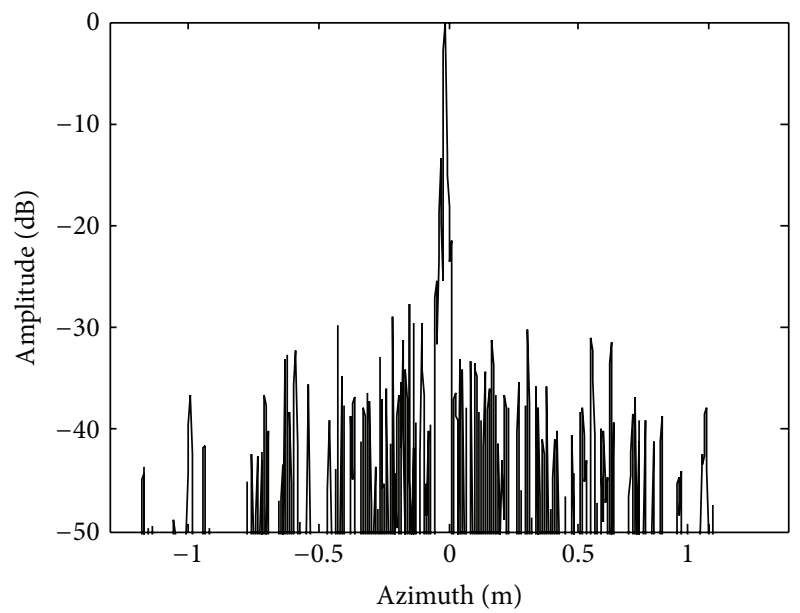

(b) DPCA SAR imaging with proposed CS method

FIGURE 13: Experimental results of azimuth profile.

\section{Conclusion}

In this paper, DOA estimation from multiple-elevationchannel echoes and adaptive beamforming in real time are proposed, which can effectively solve elevation pattern loss 
problems in HRWS SAR system. In low SNR, the joint use of multiple azimuth observation data can ensure the correct estimation of DOA. HRWS SAR's one aperture transmitting and several apertures receiving mode caused nonuniform sampling in azimuth and further brought azimuth ambiguity. Our proposed method based on CS can effectively suppress this ambiguity when precise observation models are established according to systems and platforms parameters by solving an optimization problem.

\section{Conflict of Interests}

The authors declare that there is no conflict of interests regarding the publication of this paper.

\section{Acknowledgment}

This work is supported by the National High Technology Research and Development Program of China under Grant No. 2012AA121305.

\section{References}

[1] A. Currie and M. A. Brown, "Wide-swath SAR," IEE Proceedings F: Radar and Signal Processing, vol. 139, no. 2, pp. 122-135, 1992.

[2] G. Krieger, M. Younis, N. Gebert et al., "Advanced concepts for high-resolution wide-swath SAR imaging," in Proceedings of the European Conference on Synthetic Aperture Radar (EUSAR '10), Aachen, Germany, June 2010.

[3] M. Suess and W. Wiesbeck, "Side-looking synthetic aperture radar system," US Patent, 6870500, 2005-03-22.

[4] C. Fischer, C. Heer, G. Krieger, and R. Werninghaus, "A high resolution wide swath SAR," in Proceedings of the European Conference on Synthetic Aperture Radar (EUSAR '06), Dresden, Germany, May 2006.

[5] F. Bordoni, M. Younis, E. M. Varona et al., "Performance investigation on scan-on-receive and adaptive digital beamforming for high-resolution wide-swath synthetic aperture radar," in Proceedings of the Annual Dynamic Women in Business Conference, pp. 114-121, Women's Student Association, Berlin, Germany, February 2009.

[6] E. Makhoul Varona, Adaptive digital beam-forming for highresolution wide-swath synthetic aperture radar [M.S. thesis], 2011.

[7] G. Krieger, N. Gebert, and A. Moreira, "Unambiguous SAR signal reconstruction from nonuniform displaced phase center sampling," IEEE Geoscience and Remote Sensing Letters, vol. 1, no. 4, pp. 260-264, 2004.

[8] N. Gebert, G. Krieger, and M. A. Moreira, "Digital beamforming on receive: techniques and optimization strategies for highresolution wide-swath SAR imaging," IEEE Transactions on Aerospace and Electronic Systems, vol. 45, no. 2, pp. 564-592, 2009.

[9] D. L. Donoho, “Compressed sensing," IEEE Transactions on Information Theory, vol. 52, no. 4, pp. 1289-1306, 2006.

[10] E. J. Candès, J. Romberg, and T. Tao, "Robust uncertainty principles: exact signal reconstruction from highly incomplete frequency information," IEEE Transactions on Information Theory, vol. 52, no. 2, pp. 489-509, 2006.
[11] L. C. Potter, E. Ertin, J. T. Parker, and M. Çetin, "Sparsity and compressed sensing in radar imaging," Proceedings of the IEEE, vol. 98, no. 6, pp. 1006-1020, 2010.

[12] M. Younis, Digital Beam forming for High-Resolution Wide Swath Real and Synthetic Aperture Radar, Karlsruhe University, Karlsruhe, Germany, 2004.

[13] Q. Fan, X.-D. Lu, P. Zhang, and M. Xu, "Study of nonuniform azimuth sampling of DPCMAB technique in spaceborne SAR," Journal of Electronics and Information Technology, vol. 28, no. 1, pp. 31-35, 2006.

[14] S. D. Babacan, R. Molina, and A. K. Katsaggelos, "Bayesian compressive sensing using laplace priors," IEEE Transactions on Image Processing, vol. 19, no. 1, pp. 53-63, 2010.

[15] I. G. Cumming and F. H. Wong, Digital Processing of Synthetic Aperture Radar Data: Algorithms and Implementation, Artech House, Norwood, Mass, USA, 2005. 

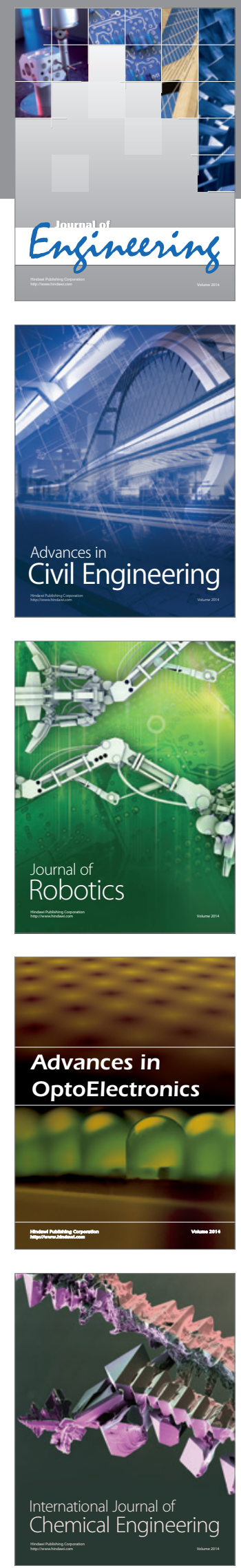

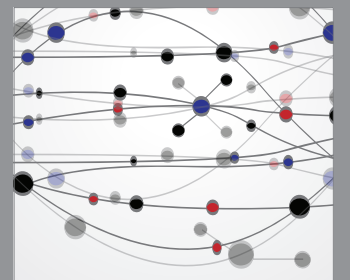

The Scientific World Journal
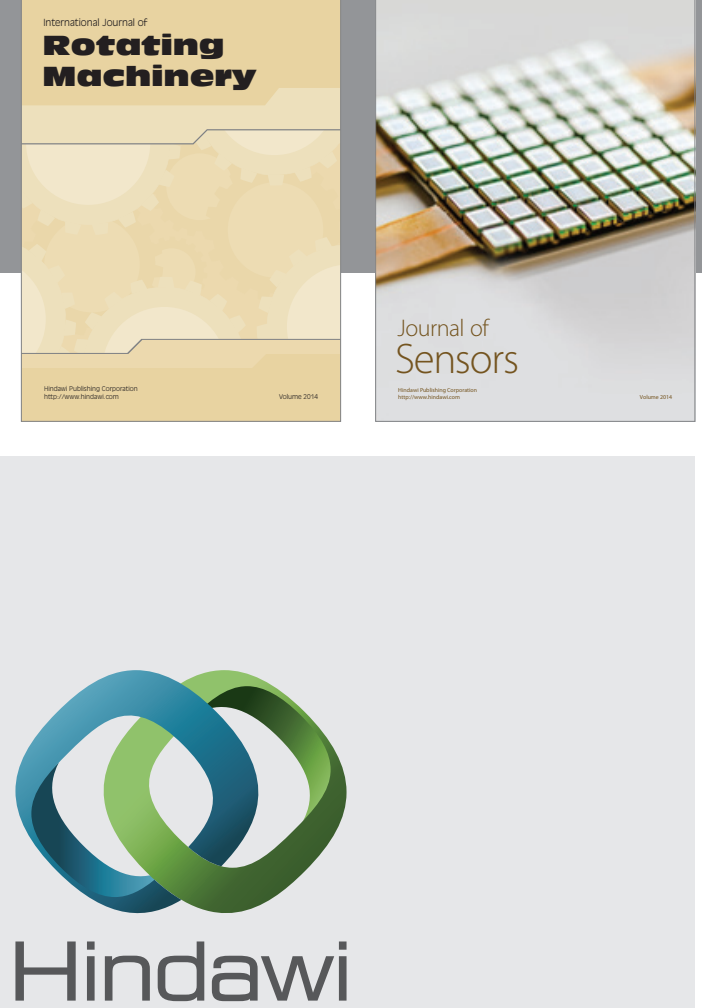

Submit your manuscripts at http://www.hindawi.com
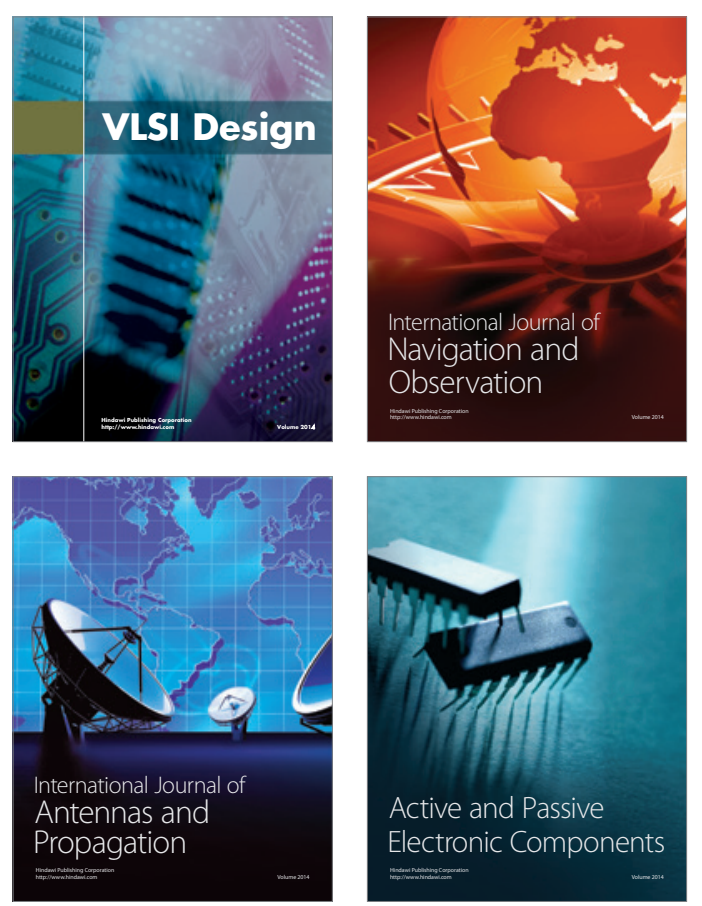
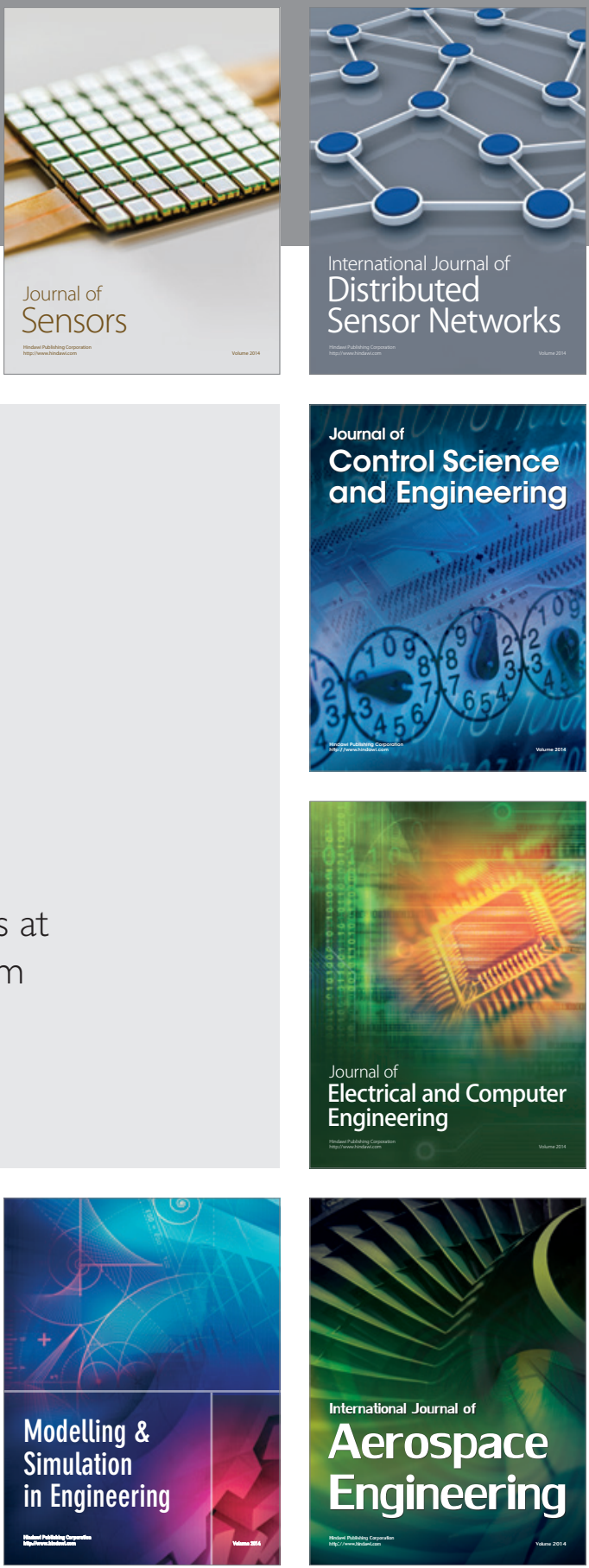

Journal of

Control Science

and Engineering
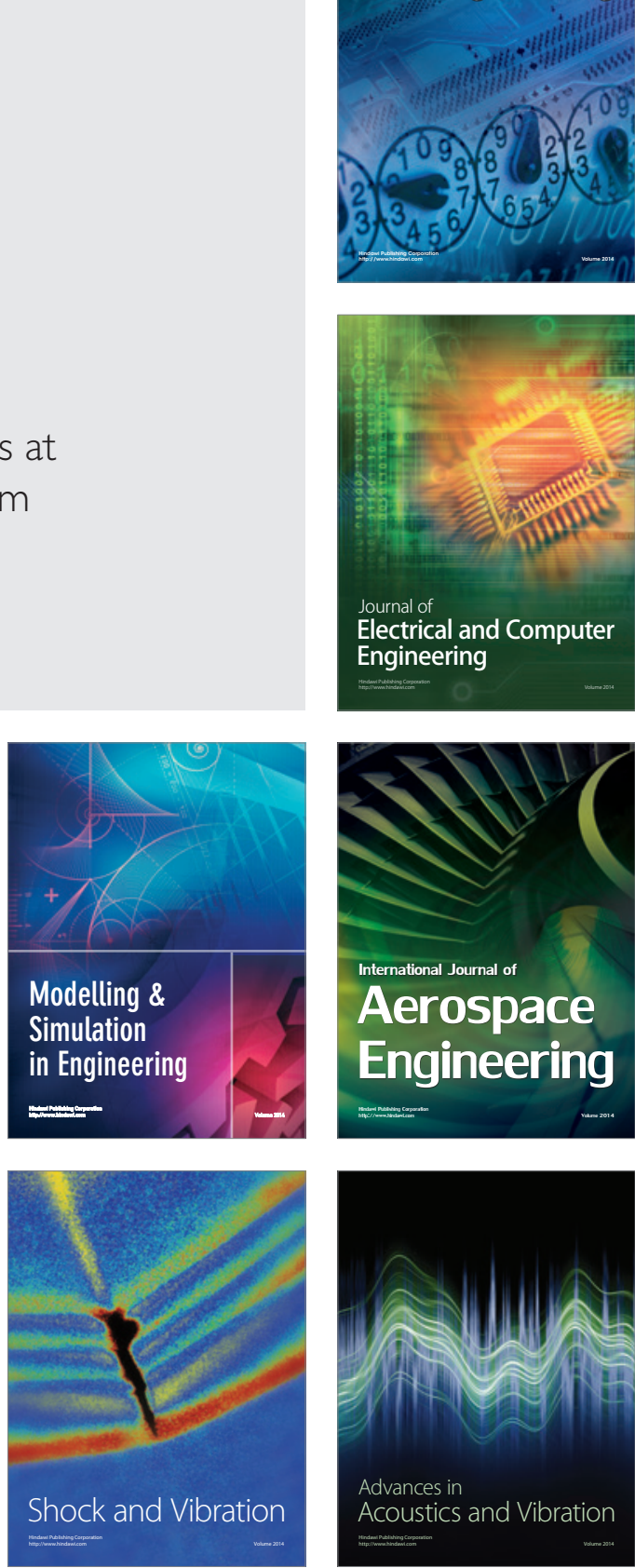